\title{
Numerus clausus in der Medizin - werden die Richtigen ausgewählt für Studium und Beruf?
}

Seit 1998 kommt an den Universitäten Basel, Bern, Freiburg und Zürich ein Numerus clausus (NC) und als Kriterium der Eignungstest für das Medizinstudium (EMS) für die Zulassung zum Medizinstudium zur Anwendung. Im Beitrag wird dargestellt, welche Faktoren einen NC früher und heute begründen, wie die Anforderungen des Medizinstudiums sich im Test wiederfinden, welche Rolle Neigungen und soziale Kompetenzen spielen und was für die zukünftige Entwicklung zu beachten ist.

Klaus-D. Hänsgen

Korrespondenz:

Prof. Dr. Klaus-D. Hänsgen Zentrum für Testentwicklung

Universität Freiburg Route d'Englisberg 9 CH-1763 Granges-Paccot ztd@unifr.ch
Immer wieder gibt es Diskussionen, ob das gegenwärtige Auswahlverfahren für den Numerus clausus in der Medizin angemessen ist, ob sich die Richtigen zum Medizinstudium anmelden oder jene gar «abgeschreckt» werden, ob die Auswahl diejenigen benachteiligen könnte, die beispielsweise Hausarzt oder Psychiater werden wollen usw.

Was kann man dazu anmerken? Es gibt zwei Forderungen, die bei jeder Studierendenauswahl zu beachten sind:

- Die gesetzlichen Grundlagen [1] des Numerus clausus sind kantonal geregelt, und in allen Gesetzen steht sinngemäss gleichlautend, dass die Eignung zum Studium bei der Zulassung zu berücksichtigen ist.

- Die Urteile des Bundesgerichts zum NC [2], das diesen guthiess (aber gesetzliche Grundlagen forderte), formulierten zwei wichtige Bedingungen: Rechtsgleichheit und Willkürfreiheit.

\section{Der NC in der Schweiz ist eine politische Entscheidung}

Wenn aktuell nur noch etwa 50\% der Angemeldeten für Humanmedizin an einer Universität mit NC einen Studienplatz erhalten, steht das zwar für eine «Verschärfung» des NC in der Schweiz - ist im internationalen Massstab aber immer noch vergleichsweise moderat. Medizin ist eine begehrte und offenbar als zukunftssicher eingeschätzte Studienrichtung mit zunehmender Nachfrage. Das «Image» des Medizinstudiums ist, dass es ein anspruchsvolles Studium ist und auch gutentwickelte Fähigkeiten zur Wis-
Numerus clausus en médecine: sont-ce les bons que I'on choisit pour les études et la profession?

Un numerus clausus (NC) est introduit depuis 1998 aux universités de Bâle, Berne, Fribourg et Zurich, ainsi qu'un test d'aptitude en tant que critère d'admission aux études médicales en Suisse. L'article évoque quels sont les facteurs qui justifiaient un NC autrefois et le justifient aujourd'hui, de quelle manière les exigences des études de médecine se reflètent dans le test, quel rôle jouent les prédispositions et les compétences sociales des candidats et quels sont les développements que l'on peut attendre à l'avenir.

sensaneignung erfordert. Würde dieses Image verändert, dann verändern sich vermutlich auch die Bewerberzusammensetzung und die Nachfrage grundsätzlich.

Für eine «Elitenbildung» bereits bei der Anmeldung zum Studium gibt es allerdings keine Belege. Seit der Einführung des EMS 1998 haben die Anmeldungen für Humanmedizin um 112\% an den Universitäten mit NC zugenommen. Das «Leistungsspektrum» der am EMS teilnehmenden Personen wurde aber nicht besser, sondern weist zwischen den Jahren gleiche Verläufe und Variabilitäten auf. 
Die Gründe für die Einführung des Numerus clausus dürfen nicht vergessen werden. Die Universitäten waren im ersten Semester stark überlastet, die Studienbedingungen katastrophal und mit Sicherheit nicht im gewünschten Sinne persönlichkeitsbildend.

Es gab hohe Abbrecherquoten, und viele Personen wurden durch Prüfungen «selegiert», um die klinische Ausbildung noch sinnvoll durchführen zu können - deren Kapazität wegen der Patientenzahlen und Betreuungskapazitäten der Universitätskliniken damals wie heute nicht beliebig erhöht werden kann.

Seitdem hat die Zahl der ihr Studium erfolgreich abschliessenden Personen zugenommen. Ein NC erhöht bei konstanter Kapazität die Absolventenzahlen. Einige Universitäten konnten überhaupt erst nach Einführung des Numerus clausus durch die Verbesserung der Betreuungsverhältnisse Studienreformen (problemorientierter Unterricht, früherer Patientenkontakt usw.) einführen und dadurch die Persönlichkeitsentwicklung günstiger gestalten. Man sollte diese heute als normal empfundenen Verbesserungen nicht vergessen.

Der NC in der Schweiz ist eine politische Entscheidung, eine Interessenabwägung zwischen einer bezahlbaren Ausbildungsqualität und der Studiennachfrage - nicht gedacht als berufspolitisches Regelungsinstrument. Je «schärfer» der NC wird, um so mehr gilt, dass einige der Abgelehnten das Medizinstudium sicherlich auch bewältigt hätten. Es muss gewährleistet sein, dass die tatsächlich Zugelassenen es besser bewältigt haben und z.B. den Abschluss schneller und mit besseren Ergebnissen erreicht haben. Ausbildungskapazitäten und deren Festlegung sind ein politisches Thema. Ärztebedarf und Studienplatzangebot müssen so in Übereinstimmung gebracht werden, dass es für die Kantone auch bezahlbar bleibt. Die Ärztedichte hat sich ja nicht vermindert, sondern es scheint ein «Strukturproblem» zu geben, indem einzelne Versorgungsbereiche für die Absolventen weniger attraktiv sind. Wenn die Nachfrage nach einem Medizinstudium wie bisher bleibt oder weiter steigt, werden auch im Falle von Anhebungen der Ausbildungskapazitäten weiterhin ein $\mathrm{Nu}$ merus clausus und damit ein funktionierendes Zulassungskriterium notwendig sein. Beim Vorschlag des SWTR, die Kapazität um 20\% anzuheben [3], stünden für 2007 dann 2000 Anmeldungen immer noch nur 700 Plätze in Humanmedizin gegenüber. Eine neue Mobilität innerhalb Europas bei der Wahl des Studienortes stellt neue Anforderungen für eine Sicherung der
Ausbildungskapazitäten gegen Überlastung (was sich z. B. in Österreich als Problem zeigt). Auch die Schweiz wird hier vermutlich langfristig mehr Nachfrage erhalten.

\section{Weshalb wird der EMS mit Schwerpunkt auf naturwissenschaftlichen Fähigkeiten durchgeführt?}

Die Gesetze der Kantone schreiben vor, dass die Studieneignung bei der Auswahl zu berücksichtigen ist. Die hohen Abbruchquoten waren vor allem auf fehlende Kompetenzen auf diesem Gebiet zurückzuführen. In den ersten Jahren des NC wurden nur wenige Personen praktisch abgewiesen und im Nachrückverfahren dennoch zugelassen. Diese Personen haben in der ersten und zweiten Vorprüfung deutlich schlechtere Leistungen erreicht bzw. sind gar nicht angetreten der Test hat diese geringen Erfolgsaussichten vorhergesagt [4]. Es sollen diejenigen bevorzugt einen Studienplatz erhalten, die die grösste Chance haben, das Studium auch zu beenden. Dies spart nicht nur Kosten für Studienplätze, die Vergeudung von individuellen Ressourcen ist ebenso bedeutsam, wenn Studierende Lebenszeit erfolglos auf diesem Weg verbracht haben.

Der EMS sagt diese Studieneignung gemessen an Massstäben vergleichbarer Tests sehr gut voraus (siehe Evaluationsberichte zum EMS [4]): Je höher das Testergebnis, um so wahrscheinlicher ist das Bestehen der Prüfung. Die Zulassung aufgrund des Testwertes ist also ausreichend begründet. Die seinerzeitigen Anforderungsanalysen für ein Studium der Medizin wurden sehr aufwendig und von verschiedenen Seiten durchgeführt [5]. Auf der Grundlage dieser Analysen wurden etwa 50 Bereiche und Aufgabentypen geprüft und die unter vielen Aspekten am besten geeignet erscheinenden 13 für die Erprobungen im Übergangsverfahren des TMS ausgewählt und empirisch erprobt. Von diesen haben aufgrund insbesondere der Validitätsergebnisse dann 9 Aufgabengruppen Eingang in den TMS gefunden.

\section{Erfassung der sozialen Kompetenz}

Für das Auswahlverfahren entsteht erst dann neuer Handlungsbedarf, wenn sich die Studienanforderungen ändern würden. Dies wird kontinuierlich beobachtet. 2003 fand dazu z.B. ein Workshop mit den beteiligten Fakultäten statt, und ein Ergebnis ist, dass die Planungskompetenz durch einen neuen Untertest «Planen und Organisieren» seit 2005 erfasst wird. Eine nächste Runde wird aktuell geplant. 
Soziale Kompetenzen sollen grundsätzlich auch erfasst werden - hier gibt es zwei Probleme:

- Sie gehen nicht bis kaum in die Bewertungen (Notengebung) beim Studium und damit den Studienerfolg ein.

- Alle bisher betrachteten Tests zur «sozialen Kompetenz» sind trainier- und damit verfälschbar. Man kann sich relativ leicht in Fragebögen als sozial kompetent beschreiben, wenn man die Konstruktionsprinzipien ungefähr kennt. Auch Interviews können nicht für 2000 Personen vergleichbar objektiv durchgeführt werden. Nur ganz wenige Testkonzepte, die mehr Wert auf die Erfassung sozialer Fähigkeiten legen, sind eventuell geeignet.

Die Erfassung der sozialen Kompetenz bleibt eine methodische Herausforderung für die $\mathrm{Zu}$ kunft.

Man kommt weiter, wenn man Eignung und Neigung unterscheidet. Neigung wäre alles, was mit Interesse und Motivation für ein Studienfach umschrieben werden kann. Bei fehlender Neigung wird ein Studium sicher gar nicht aufgenommen. Problematischer sind die Fälle, bei denen man während des Studiums entdeckt, dass ein Fach nicht den eigenen Neigungen entspricht. Dies kann an fehlenden oder falschen Informationen über Studium und Beruf liegen. Die Erfassung von Neigungen und Verwendung als Zulassungskriterium ist wegen der hohen Anfälligkeit für Trainierbarkeit und Verfälschung («sich im besten Licht darstellen») ebenfalls praktisch unmöglich.

\section{Informationsmöglichkeiten zum Beruf}

2006 und 2007 wurden die EMS-Teilnehmer befragt, und nur $14 \%$ erlebten die Informationsmöglichkeiten zum Wunschberuf in beiden Jahren als zu gering oder viel zu gering, der Rest als ausreichend bis sehr gut. Die Informationsmöglichkeiten zu den Studienanforderungen erlebten $17 \%$ (2006) bzw. 16\% (2007) als zu gering oder viel zu gering. Entweder es gibt wirklich genügend Informationen - oder das vorhandene Informationsbedürfnis vor dem Studium wurde im wesentlichen gestillt, auch wenn es zu niedrig wäre. Die Studierenden müssen die Anforderungen des Studiums und des Berufes vor einer Bewerbung besser kennenlernen und sich z. B. auch damit auseinandersetzen, was ein Hausarzt tut und ob man sich dazu berufen fühlt. Eine Fachrichtung wird nur gewählt, wenn sie attraktiv ist und den eigenen Neigungen entspricht.
Eine in anderen Ländern erfolgreich eingesetzte Methode sind hierfür Studienberatungsinstrumente, die zur «Selbstberatung» angeboten werden und meist im Internet realisiert sind. Es werden detaillierte Informationen über die verschiedensten Anforderungen von Studium und späterem Beruf gegeben und Checklisten sowie Tests angeboten, um ohne Druck eigene Fähigkeiten und Neigungen zu erkunden. Da hier das Interesse der Personen an realistischen Aussagen überwiegt (weil es nicht als Zulassungskriterium verwendet wird), ist es auch nicht sinnvoll, sich in einem «besseren Licht» darzustellen. Es gibt mittlerweile Universitäten, welche die Absolvierung eines solchen Studienberatungstestes zur Pflicht machen (ohne die Ergebnisse selbst für die Zulassung zu verwenden). Es wäre eine Herausforderung mit grossem erwartetem Nutzen, für Medizin ein solches Selbstberatungsinstrument zu entwickeln - die meisten und bekannten Instrumente beziehen sich bisher auf technische Berufe.

\section{Wer interessiert sich \\ für das Fachgebiet «Hausarzt»?}

Die Berufsinteressen der Teilnehmenden am EMS werden seit 2005 jährlich nach dem Test in einem freiwillig auszufüllenden Fragebogen mit erfragt. Es sind «nur» jeweils um 10\% aller Testteilnehmer in der Schweiz, die Hausarzt werden wollen. Die Anteile decken sich mit den Ergebnissen der drei Quoten in Österreich 2006 (Abb. 1). Das ist offenbar eine sehr stabile Ausgangslage, wo sich andeutet, dass die «Hausarztdiskussion» in der Schweiz einen minimalen Effekt zeigt. Diese Ausgangssituation kann man nur ändern, wenn weiter gezielt für die Orientierung «Hausarzt» geworben wird - und man im Auge behält, dass die Entscheidung nach dem Studium eine ganz andere sein kann, wenn man die Fächer genauer kennt.

\section{Was ist über die Interessenten für «Hausarzt» bekannt?}

Es zeigen sich über die letzten drei Jahre sehr konsistente Ergebnisse: 2005 lag der mittlere Testwert bei 100,4 (30 Männer: 101,6; 68 Frauen: 99,9). 2006 betrug der mittlere Testwert 100,6 (68 Männer: 101,1; 101 Frauen: 100,3). 2007 war der mittlere Testwert 101,0 (56 Männer: 101,8; 95 Frauen: 100,5).

- Die Bewerber für «Hausarzt» erreichen keine schlechteren Ergebnisse als andere (der Testwert hat für alle Kandidaten bekanntlich den Mittelwert 100). 


\section{Abbildung 1}

Ergebnisse der Befragungen zum zukünftig gewünschten Fachgebiet (nur Bewerbungen Humanmedizin). Schweiz 2005 bis 2007 und Vergleichsdaten Österreich 2006 für die Quoten «EU-Bürger» (AT-EU), «Bürger mit österreichischer Maturität» (AT-AT) und «Nicht-EU-Bürger» (AT-NONEU) (in Klammern die Personenzahl).

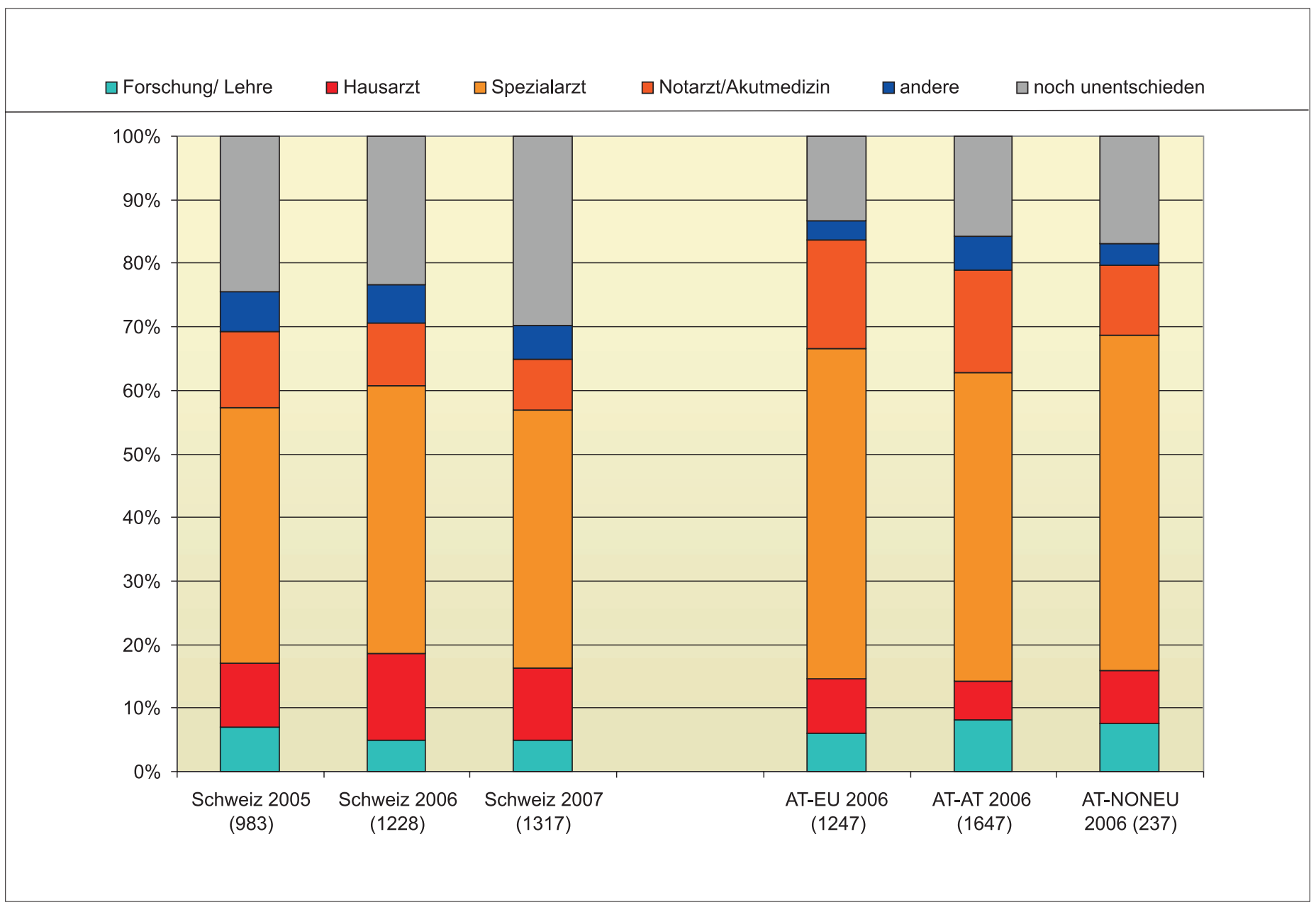

- Das Geschlechterverhältnis weicht nicht wesentlich vom Verhältnis in der Gesamtgruppe (60\% Frauen, $40 \%$ Männer) ab.

- Auch der Testwertunterschied Männer zu Frauen liegt im Rahmen der Unterschiede der Gesamtgruppe.

Interessenten für «Hausarzt» sind also ganz «typische» Bewerber für ein Medizinstudium und überhaupt nicht schlechter im naturwissenschaftlichen Denken. Sie werden bei der Zulassung nicht benachteiligt. Insofern ist auch die Annahme, dass Interessenten für den Hausarztberuf naturwissenschaftlich «minderbemittelt» wären, unbegründet.

Auch das fehlende Interesse für Psychiatrie scheint eher ein «Neigungsproblem» zu sein. Die «antipsychiatrische» Diskussion lebt in der Öffentlichkeit momentan ziemlich polemisch auf, und der Berufsstand steht unter Kritik, ob es sich bei Psychiatrie um eine seriöse Wissenschaft handelt oder nicht. Wäre es nicht wichtiger, die Integration dieses Faches als vollwertiges Fach der Medizin zu betonen, statt eine Trennung der Ausbildung vorzunehmen, die dann im Verdacht steht, auch eine Abgrenzung zu sein? Neigung und damit Nachfrage wird so nicht gefördert, und es werden sich noch weniger Studierende für dieses Fach interessieren.

Immer wieder wird auch die Forderung gestellt, Elemente der Berufseignung im Sinne des «guten Arztes» mit in den Zulassungstest aufzunehmen. Sehen wir einmal davon ab, dass die aktuellen gesetzlichen Grundlagen eine Beschränkung auf Studieneignung vorschreiben: Was bliebe offen? Die nichtkognitiven Voraussetzungen oder «social skills» (Empathie, soziale Kompetenz usw.) sind vermutlich für die einzelnen Fachgebiete ganz unterschiedlich zu gewichten, sind wenig analysiert und auch durch Testverfahren nicht mit der gleichen Güte zu messen wie die kognitiven Voraussetzungen. 
Letztendlich muss der Medizinstudierende auch hier «nur» bestimmte Fähigkeiten mitbringen, die inhaltliche Ausformung und Differenzierung ist Aufgabe des Studiums und eigene Neigungen und Interessen werden die Wahl des Fachgebietes dann mitbestimmen. Insofern wäre es vielleicht ausreichend, soziale Kompetenzen als soziale Fähigkeiten in geeigneter Weise mitzuerfassen und dann der Ausbildung zu überlassen, dass diese Fähigkeiten auch umgesetzt werden.

Der EMS ist kein Wissenstest, sondern ein Fähigkeitstest. Es werden keine Fakten abgefragt, sondern Problemstellungen mit den notwendigen Informationen vorgegeben, die zu lösen sind. Intendiert ist, dass es sich um eine «Arbeitsprobe» für die Studierfähigkeit handelt. Es wäre vielen Menschen wichtig, dass der behandelnde Arzt auf der Basis bester biologisch-naturwissenschaftlicher Grundlagen und einschlägig fundierten Wissens handelt - und nicht nur empa- thisch-intuitiv und begründet auf privater Erfahrung. Die Fähigkeit zum Wissenserwerb braucht man lebenslang.

\section{Literatur}

1 www.crus.ch/information-programme/ anmeldung-zum-medizinstudium.html.

2 Urteile des Schweizerischen Bundesgerichtes 2P.171/1998/mks vom 3. März 1999 sowie 2P.228/1998 - 2P.332/1998/luc vom 20. August 1999.

3 Ärztedemographie und Reform der ärztlichen Berufsbildung. Bericht des SWTR vom 6.9.2007.

4 Hänsgen K-D, Spicher B. Numerus clausus: Finden wir mit dem «Eignungstest für das Medizinstudium» die Geeigneten? Schweiz Ärztezeitung. 2002; 83(31):1653-60.

5 Trost G, Blum F, Fay E, Klieme E, Maichle U, Meyer M, Nauels H-U. Evaluation des Tests für Medizinische Studiengänge (TMS): Synopse der Ergebnisse. Bonn: ITB; 1998. 